\title{
Los consumos juveniles de música en la era digital: un estudio de caso en la Zona Metropolitana de Querétaro
}

\author{
YOUTH CONSUMPTION OF MUSIC IN THE DIGITAL AGE: A CASE STUDY IN THE \\ METROPOLITAN AREA OF QUERÉTARO \\ CONSUMO DE MÚSICA DOS JOVENS NA ERA DIGITAL: UM ESTUDO DE CASO \\ NA REGIÃO METROPOLITANA DE QUERÉTARO
}

\section{Sergio Rivera Magos* \\ Bruno Carriço Reis**}

Cuadernos de Música, Artes Visuales y Artes Escénicas

/ Volumen 10 - Número 2 / julio - diciembre de 2015

/ ISSN 1794-6670/ Bogotá, D.C., Colombia / pp. 171-192

Fecha de recepción: 5 de mayo de 2015 | Fecha de

aceptación: 30 de agosto de 2015 | Disponible en línea:

18 de diciembre de 2015. Encuentre este artículo en

http://cuadernosmusicayartes.javeriana.edu.co/

doi:10.11144/Javeriana.mavae10-2.cjmd

* Profesor de la Universidad Autónoma de Querétaro.

* * Profesor de la Universidad Autónoma de Lisboa, investigador del Núcleo de Estudos em Arte, Media e Política (NEAMP) de la Pontifícia Universidade Católica de São Paulo. 


\section{Resumen}

Internet trajo consigo importantes cambios en el consumo y apropiación de la música. El siguiente trabajo tiene como objetivo explicar los consumos musicales de los jóvenes de la Zona Metropolitana de Querétaro y el papel que las Tecnologías de la Información y Comunicación (TIC) desempeñan en ellos. Se propone aquí un acercamiento a la música como objeto de estudio a partir de una metodología dialógica, que escucha a los jóvenes cuando hablan acerca de sus prácticas musicales. Los principales hallazgos muestran cómo las TIC han contribuido a que la música sea un elemento omnipresente en la vida de los jóvenes del estudio de caso. También explica cómo la música ha pasado de ser un producto de consumo a solamente un contenido, y cómo los jóvenes dejaron de ser consumidores, para convertirse en oyentes.

Palabras clave: música; jóvenes; consumo digital; Internet

\begin{abstract}
Internet brought along important changes in the consumption and embracement of music. The objective of the following work is to explain the musical consumptions of the youth in Querétaro's city Metropolitan Area, and the place that Information and Communication Technologies (ICT) occupy within them. Here we propose an approach to music as an object of study from a dialogic methodology, listening to the youth talk about their musical habits. The outstanding findings show how ICT have contributed in making music an omnipresent element in the life of the young people of the case study. As well, it explains how music has been transformed from a product of consume into simply content, and how young people are no longer consumers but listeners.
\end{abstract}

Keywords: music; youth (young people); digital consume; Internet

\section{Resumo}

Internet trouxe consigo grandes mudanças no consumo e apropriação musical. Este artigo visa explicar o consumo de música dos jovens da Região Metropolitana de Querétaro, levando em linha de conta o papel decisivo que desempenham as Tecnologias de Informação e Comunicação (TIC) na conformação deste fenómeno social. Propomos uma abordagem à música como objeto de estudo desde uma metodológica dialógica, ouvindo os jovens acerca das suas práticas musicais. Os principais resultados mostram como as TIC's têm contribuído para que a música seja omnipresente na vida dos jovens. Este estudo também explica como a música deixou de ser um produto de consumo para passar a ser um conteúdo e como os jovens deixaram de ser consumidores, transformando-se em ouvintes.

Palavras-chave: música; juventude; consumo digital; Internet 


\section{INTRODUCCIÓN}

Los consumos musicales juveniles se han convertido, desde la irrupción de las nuevas Tecnologías de la Información y la Comunicación (TIC), en un objeto de estudio privilegiado para entender la articulación entre música, jóvenes y nuevas tecnologías. Como afirma Fouce (2008), la música y la televisión han sido las dos principales expresiones de la cultura de masas, por ello reviste gran importancia explicar cómo en el contexto de la era digital los jóvenes se apropian de ella.

La música debe ser tomada en consideración como parte importante del consumo cultural, ya que asume un papel protagónico en la creación y difusión de significados para los jóvenes; por ello su estudio se ha convertido en un elemento fundamental para la comprensión de la manera como ellos crean identidad y conforman sus estilos de vida. Su análisis permite entender las distintas configuraciones del mundo que de manera contradictoria y compleja, los jóvenes construyen a partir de sus vínculos con el mercado de consumo y las industrias culturales (Reguillo, 2012). La música asume en el ámbito específico de los consumos culturales juveniles, un papel social relevante, como forma de creación de identidad, entretenimiento y socialización.

En esta investigación entendemos las culturas juveniles a partir de los elementos distintivos con los que Reguillo (2012) propone reconocerlas: las Tecnologías de la Información y la Comunicación (TIC), los medios masivos de comunicación, las modas en la música, la ropa, la estética, las problemáticas globales y el deterioro del planeta. De todo este catálogo de elementos y circunstancias, para esta investigación son fundamentales la música y el uso de las TIC, como marcadores juveniles.

Respecto a la importancia de las nuevas tecnologías en la configuración de culturas juveniles, Macassi sostiene que es:

(...) un proceso de desterritorialización de la cultura, donde Internet tienen una presencia dina-

mizadora, en tanto que los productos comunicativos tienden cada vez más a construir códigos

y referentes, 'comunidades hermenéuticas de consumidores' más allá de las fronteras de los

Estado-nación, así tenemos que las modas, los bailes y la música son referentes desterritoria-

lizados de los cuales los jóvenes son los abanderados. (2001, p. 34)

Esta investigación hace énfasis en la idea de que la música es un elemento simbólico y cultural del cual los jóvenes se apropian como parte fundamental de la cultura juvenil a la que pertenecen. No hay que olvidar que las culturas juveniles que emergieron en la posguerra hacia los años cincuenta del siglo XX han evolucionado vertiginosamente a partir de la aparición de las TIC; las nuevas culturas juveniles de las primeras décadas del siglo XXI son producto de las apropiaciones que han hecho de Internet y de los equipos digitales que usan en la vida cotidiana.

La música, lejos de ser algo marginal o secundario en el estudio de las culturas juveniles, es una variable central para su comprensión. Como apunta la antropóloga Ruth Finegan "más que simplemente iluminar los elementos de la cultura, constituye una dimensión fundamental para la comprensión de un grupo cultural particular" (2002, p. 16). La música debe ser entendida entonces como una manifestación propia de la condición juvenil, pero también como configuradora de esta, una expresión inherente al hecho de ser joven y manifestarlo. 
La música es algo muy importante en la vida de los jóvenes, pues, además de su uso como forma de entretenimiento y compañía, a través de ella interactúan con sus pares, comunican sus emociones, generan identidad. La relevancia de la música para los jóvenes ha sido determinada empíricamente en diversos estudios (Green, 1997; Gasperoni, Marcon y Santoro, 2004; North, Hargreaves y O’Neil, 2000), y como bien apunta el sociólogo de la música Simon Frith a propósito de la juventud en el Reino Unido, (2001) la historia de las audiencias del pop británico es siempre una historia de los estudios de los jóvenes británicos.

Además de la música, otro elemento importante para la configuración de lo juvenil es la tecnología. Según Susana Reguillo:

Las tecnologías en sus diferentes vertientes operan como conectores, prótesis, plataformas, catapultas, experiencia cotidiana para interactuar con el mundo: del plumón para graffitear una pared a la computadora con Internet que permite acceder a la producción de autoría (es decir a la voz propia) y acceso a múltiples redes sociales. La tecnología es la marca de época de una juventud que la utiliza tanto para afirmar sus pactos con la sociedad de consumo, como para marcar sus diferencias y críticas a esa sociedad. $(2012$, p. 1)

Cada generación ha estado marcada por la tecnología a su alcance y por la forma de utilizarla. El consumo de música se ha beneficiado de la tecnología a lo largo del tiempo en cuanto a los cambios respecto a la portabilidad, la miniaturización y la inmaterialidad. Los jóvenes de las diferentes generaciones han consumido música y la han usado para diversos fines, lo cual hace de la tecnología una variable crucial en la configuración de sus prácticas de consumo musical.

La intersección de los dos aspectos antes mencionados, y la centralidad de la música en las prácticas juveniles mediadas por un fuerte proceso tecnológico, nos propuso la formulación de un estudio que respondiera a la pregunta: ¿Los actuales consumos musicales juveniles presentan renovadas formas de apropiación mediante la utilización intensiva de las TIC? Así mismo, que tuviera como objetivo general comprender los consumos de música de los jóvenes del municipio de la Zona Metropolitana de Querétaro (ZMQ). Dicha comprensión permitiría además acceder a los objetivos específicos: a.-Describir el uso de las TIC en el consumo de música por parte de los jóvenes de la ZMQ; b.- Interpretar las prácticas de consumo de música por parte de los jóvenes de la ZMQ.

La respuesta a la pregunta y el cumplimiento de los objetivos mencionados requerían una discusión teórica concebida para articular los diferentes aportes de las disciplinas de la teoría social acerca de la música, con el objetivo de llegar a un modelo único para aplicarlo empíricamente. Para ello se hizo un mapeo que nos llevó de la musicología a la sociología de la música, y de ahí a las ciencias de la comunicación, en donde identificamos los aportes y limitaciones de cada disciplina, con la intención de llegar a un modelo de estudio adecuado para el análisis de los consumos y usos juveniles de la música.

La articulación llevada a cabo en la investigación parecía pertinente, dado que el campo del estudio de la música ha sido difícil y controversial, pues aún hoy en día persiste la pregunta de si la música es un objeto de estudio autónomo o si más bien debería estar comprendido dentro de otras disciplinas como la sociología de la cultura. La aproximación teórica pretendía poner en evidencia la necesidad de estudiar a la música como un objeto de estudio en sí mismo, echando mano de diferentes herramientas provenientes de la sociología, la antropología y las ciencias de la comunicación. 
El trabajo aquí presentado comienza por plantear un estado de la cuestión acerca del fenómeno musical y sus diferentes formas de ser abordado, desde la musicología hasta la sociología de la música, para llegar finalmente al encuentro de música y ciencias de la comunicación a través de los estudios de recepción y sus diferentes temas de investigación dentro del ámbito musical. El segundo segmento de este artículo pretende reseñar el recorrido histórico por los procesos de transformación del oyente juvenil; es decir, cómo la apropiación musical juvenil se va transformando a partir del impacto de las nuevas tecnologías, y genera nuevos usos y concepciones de la música.

En el apartado acerca del diseño metodológico se explican las particularidades del estudio, su carácter cualitativo y la importancia de la Zona Metropolitana de Querétaro, México, como un área especialmente propicia para el análisis de los consumos musicales juveniles. Así mismo, se explican los criterios para la elección de la muestra, sus particularidades y el uso del espacio público para abordar a los jóvenes consumidores de música que fueron entrevistados. Como parte de la presentación de la estrategia metodológica se propone el uso de la entrevista semi-estructurada como técnica principal y se explica puntualmente la naturaleza, la articulación del modelo de análisis, sus conceptos y dimensiones.

En la exposición de los principales resultados se explica que la información empírica con la que se sustentan los diferentes argumentos y afirmaciones fue tomada de un proyecto de investigación mayor, del cual se usaron los datos relacionados con el uso de YouTube por parte de los jóvenes de la muestra. En esta sección se presentan los hallazgos más significativos de acuerdo a las diferentes categorías analizadas haciendo énfasis en la importancia de los teléfonos móviles en el consumo juvenil de música en el contexto de la vida cotidiana. Por último, se presentan las conclusiones producto de la contrastación de la propuesta teórica con el análisis de la información recabada durante el trabajo de campo con los jóvenes consumidores de música de la Zona Metropolitana de Querétaro.

\section{APROXIMACIÓN TEÓRICA: ESTADO DE LA CUESTIÓN DEL FENÓMENO MUSICAL}

El estudio de la música ha transitado por diferentes etapas en las que se destaca algún elemento o género en particular. Del estudio exclusivo de la música culta occidental a la fascinación por el folclor, de ahí al estudio de la música popular y luego al de la música urbana, la música ha sido un lugar de encuentro entre diferentes disciplinas, pero es en la sociología de la música y las ciencias de la comunicación, en los estudios de recepción, donde el consumo de música popular recibe una mayor atención y se visibiliza. Por ello es importante una revisión de los aportes de estas áreas en cuanto al tema musical y los diferentes enfoques con que lo estudiaron.

El estudio de la música comienza con la musicología, "disciplina diseñada para observar y estudiar académicamente la organización del sonido musical y las actividades a que da lugar" (Rodríguez, 2008, p. 10). El academicismo y su orientación hacia el estudio de la música clásica fueron limitantes que evidenciaron la necesidad de nuevas disciplinas que pudieran abrir el estudio de la música a otros enfoques, y sobre todo a otros sonidos. Es entonces cuando llega la etnomusicología como una respuesta a la necesidad de estudiar la música con un hecho cultural, más que como fenómeno sonoro. Figuras como Alan Merriam (1964) Ilevaron la etnomusicología a prácticamente fusionarse con la antropología de la música, al punto que suele usarse estas nomenclaturas indistintamente. 
Al mimetizarse con la antropología y tomar de ella sus principales herramientas metodológicas, la etnomusicología da un paso adelante hacia la independencia de la música como objeto de estudio y la libera del yugo estético que la musicología imponía a su estudio, pudiendo dejar atrás la obra clásica para abrirse a la música de otro origen y denominación. Los estudios provenientes de la etnomusicología aportan muchos datos sobre la dimensión cultural del comportamiento musical, pero como sostiene Steingress (2008, p. 237) "no incluyen los aspectos nucleares del análisis sociológico." Estas omisiones hacían imperativo generar una disciplina que se ocupara de la dimensión social que la etnomusicología no estaba explorando. La etnomusicología se constituiría en el puente entre la musicología y la sociología de la música, disciplina menos difusa que acaba por integrar los esfuerzos por dirigir el análisis de la música hacia su dimensión socio-cultural.

\section{LA SOCIOLOGÍA DE LA MÚSICA: ENTRE LA RACIONALIDAD Y LA SOCIALIZACIÓN}

A partir de la obra de los sociólogos alemanes George Simmel (2003) y MaxWeber (1921), la sociología empieza a reconocer a la música como objeto de estudio. Surge el interés por saber cómo los individuos se apropian de ciertas músicas y cómo las utilizan en la vida cotidiana, para lo cual plantean como imprescindible estudiar los usos y funciones de la música. Weber establece un paralelismo entre el desarrollo de la sociedad y el de la música, además reconoce a la tecnología como uno de los elementos nodales en el estudio de la misma, lo que sentó las bases para que actualmente la tecnología sea una de las principales líneas de investigación de la sociología de la música.

Heredero de la teoría marxista, Theodor. W. Adorno y su obra constituye un nuevo giro de tuerca para la sociología de la música. Su principal legado es la Filosofía de la nueva música (1949). Si bien Adorno reconoce la relación entre música y sociedad, también aclara que la música es en buena medida autónoma y tiene una dinámica considerablemente más compleja de lo que propone la teoría marxista. Su preocupación no se reduce a la preservación del valor estético de la música y la independencia creativa del artista, también le preocupa que la cultura de masas la despoje de su carácter revolucionario y su verdad intrínseca. Con base en sus ensayos surgieron otros enfoques que trataron de trascender la visión estética de la música para explorar su dimensión cultural.

A partir de los aportes y limitaciones de la teoría musical de Adorno, los estudios culturales en Inglaterra (1964) centran su atención en la cultura popular permitiendo estudiar géneros musicales como el rock, el punk o el heavy metal, los que se convierten en atractivos territorios simbólicos desde donde estudiar la cultura y la vida cotidiana de los jóvenes británicos. El estudio sociológico de la música contemporánea encontró en los estudios culturales una herramienta eficaz para explorar aspectos de la música ignorados repetidamente por la musicología. Simon Frith (1980) sostiene que a partir de esta atención a la cultura popular, es posible analizar dos preocupaciones básicas: el significado de la cultura de masas y el estudio de las culturas juveniles. ${ }^{2}$

Los estudios culturales, tanto en sus aportes como a partir de la crítica que se hizo de sus planteamientos, permitieron avanzar sobre nuevas formas de observar el fenómeno y las problemáticas derivadas de la música. En Latinoamérica por ejemplo, se comenzó a estudiar la música popular, y especialmente el rock, utilizando los conceptos de campo y habitus de 
Pierre Bourdieu, autor que se ha convertido en un referente obligado para los investigadores del consumo cultural en general, y del musical en particular. En uno de sus libros paradigmáticos La distinción: criterios y bases sociales del gusto (1979), el sociólogo francés analizó profusamente el elitismo de la música culta, y el gusto como una creación social. Si bien es cierto que explicar cómo se configura el gusto musical juvenil implica necesariamente recurrir al habitus bourdiano, para poder entender el papel de la clase social y/o la educación en la construcción de las preferencias musicales, la llegada de Internet también implica repensar el impacto de estas variables.

\section{EL ANÁLISIS DE LA MÚSICA DESDE LOS ESTUDIOS DE RECEPCIÓN: RETOS Y OPORTUNIDADES}

Aunque la música no ha sido uno de los objetos de estudio más atendidos por las ciencias de la comunicación, tienen diversos puntos de encuentro, y los estudios de recepción es uno de ellos. No podemos comenzar con la mención de trabajos o esfuerzos en este ámbito sin reconocer que el estudio de los usos y funciones de la música ha representado un reto teóricometodológico para las ciencias de la comunicación, dados los límites de la misma para poder abordarlos en toda su complejidad. La teoría de los usos y gratificaciones, por ejemplo, aunque reconoce la capacidad del receptor para elegir y usar contenidos musicales, no contempla las condiciones de recepción, el contexto, las oportunidades de acceso, ni la interacción social de los consumidores.

Los estudios culturales británicos, por su parte, pusieron el acento en la música como un componente de expresiones subculturales; no así la mayor parte de los latinoamericanos y algunos australianos, ${ }^{3}$ que sí han reconocido a la música como un objeto de estudio en sí misma. Una propuesta que parece superar estas limitaciones es la de Tia DeNora (2000), quien sugiere analizar la música más allá del esquema de emisión/recepción, concibiéndola como parte de un proceso social más que como resultado de la comprensión de la obra por parte del oyente.

DeNora propone una teoría de los sentidos y efectos de la música estudiándola etnográficamente, para entender cómo esta es apropiada en la vida cotidiana. Los estudios de la investigadora examinan cómo las personas utilizan la música en actividades diarias como: las rutinas laborales, la práctica del karaoke, las clases de aerobics o los encuentros íntimos. El trabajo de DeNora abona a la concepción de la música como un artefacto cultural complejo, como un objeto de estudio digno de ser reconocido por la ciencias sociales que lo han visto más como una variable explicativa para entender otros problemas e interrogantes; también abre el camino para estudios que no ignoren las condiciones de recepción, ni el contexto social de los agentes que se apropian de ella.

La relación de la música con los estudios de recepción ha dado una variedad de abordajes y propuestas. La música en la comunicación audiovisual, por ejemplo, ha llamado la atención de algunos investigadores en áreas como la publicidad, la televisión, el cine y actualmente en el campo de los videojuegos. El formato del video musical ha sido definitivo para el desarrollo de la música popular en las últimas décadas. El fenómeno del video clip tuvo su momento más alto en los ochenta con la aparición de MTV y el nacimiento de nuevos públicos consumidores de música e imagen (Sun y Lull, 1986). 
La música como industria generadora de contenidos musicales propios para los medios masivos y sus audiencias, también ha sido objeto de estudio de las ciencias de la comunicación. Podemos mencionar trabajos de Simon Frith (2006), Leonhard (2005) y Calvi (2006), quienes han analizado cómo la industria de la música se ha trasformado a partir de los cambios tecnológicos de cada época y cómo la aparición de nuevos soportes ha determinado las prácticas de consumo. En España los libros editados por Miguel de Aguilera, Joan E. Adell y Ana Sedeño $(2008,2010)$ sobre comunicación y música son algunos de los intentos más serios por tratar a la música desde las ciencias de la comunicación y dentro del marco de la tecnología y las audiencias; en ellos se abordan temas relacionados con la producción, el consumo y el contenido de productos derivados de la música. ${ }^{4}$ En México destaca el trabajo de María del Carmen De La Peza Casares El Bolero y la educación sentimental en México (2001), donde estudia a las audiencias del bolero y sus formas de apropiación; así como su trabajo El Rock mexicano, un espacio en disputa (2015). Esta investigación, por su parte, aporta a la comprensión del rock nacional introduciendo la noción de un espacio simbólico cuyo sentido es disputado por músicos y audiencias, por lo alternativo y por el mainstream.

La música y la comunicación están visibilizándose poco a poco como un objeto de estudio autónomo, separado de la musicología o de los estudios sobre música abordados desde la antropología. Sin embargo, siguen existiendo traslapes con otras disciplinas, lo cual ha impedido definir de manera nítida sus líneas de investigación. Tampoco se ha consolidado un cuerpo de estudios de recepción musical que pueda sugerir que existe ya la claridad sobre su abordaje o las metodologías propias para su análisis.

En el trabajo que aquí se presenta, se aborda la música desde los estudios de recepción por las siguientes razones:

a).- La música es un producto de la cultura popular, definido y configurado por su difusión mediática y por el devenir tecnológico aplicado a sus procesos de consumo, por tanto su estudio involucra de manera natural a las ciencias de la comunicación.

b).- La tecnología ha determinado los usos de los medios de comunicación en el último siglo, al igual que las prácticas de consumo de música.

c).- El difícil encuentro entre las ciencias de la comunicación y la música se ha inclinado hacia el estudio de los productos mediáticos y de sus audiencias, dejando de lado los estudios que profundizan en los usos de la música y las prácticas de consumo personalizado inherentes a los públicos de las nuevas tecnologías. Conviene entonces también discutir a continuación, algunas particularidades acerca de los jóvenes como consumidores de música.

\section{LA APROPIACIÓN MUSICAL: UN RECORRIDO HISTÓRICO POR LOS PROCESOS DE TRANSFORMACIÓN DEL OYENTE JUVENIL}

Durante los años cincuenta del siglo pasado, los jóvenes de la posguerra fueron los protagonistas de grandes sucesos en la generación de la música popular y su consumo hasta antes de la era digital; la creación del rock y el pop fueron dos de ellos. Un amplio mercado juvenil estimuló una industria volcada hacia la música popular, ${ }^{5}$ de carácter masivo y con amplio potencial de venta. Desde su conformación, la industria de la música estuvo vinculada a la tecnología disponible en ese momento. Su desarrollo se fue dando de manera paralela a las innovaciones tecnológicas. La posibilidad de fijar la música, de contenerla en un soporte, 
determinó su potencial de venta, pero también de uso, lo que generó una industria basada en la reproductibilidad de la música y la posibilidad de poseerla. La industria se construyó con base en la innovación tecnológica, y fue precisamente esta la que determinó el fin de un modelo y el surgimiento de uno nuevo basado en la música digital y su manejo.

Aunque el CD es el primer soporte propiamente digital, es la llegada de Internet la que cambia el paradigma de consumo juvenil de música, que permite la transición de los consumos analógicos a los digitales. De acuerdo con lo dicho por Reguillo (2012), Internet es la marca distintiva para esta generación y el parte aguas del mercado juvenil de música. Internet no solo es un nuevo medio, sino también un generador de nuevos modelos sociales y culturales; por ello, "entender la música y sus prácticas culturales, implica ubicar éstas en un marco más amplio, en el marco de la cultura digital" (Fouce, 2009, p. 8).

Uno de los grandes efectos de la tecnología digital en la música, fue el de convertir un producto de consumo vinculado al objeto y a los sistemas de distribución convencional, en uno basado en la información. La música digitalizada se convirtió rápidamente en un contenido, información que se puede copiar para compartirse o almacenarse en forma más rápida y práctica. La desmaterialización de la música, propia de la tecnología digital, trajo consigo: la fragmentación de los consumos a partir de canciones sueltas, el almacenamiento abundante de archivos musicales, la generalización de la escucha móvil, la descarga irrestricta, y el compartir música sin soporte a través de redes sociales y de dispositivos móviles (Carriço Reis, 2009). Internet permitió el consumo musical a través de plataformas audiovisuales que se convirtieron rápidamente en el recurso principal para la consulta y descarga de música: de manera sencilla y a través de un solo clic, YouTube por ejemplo permite satisfacer todos los apetitos musicales. La "inmediatez" se inscribe en la lógica de la velocidad y del presente continuo que caracteriza a las culturas juveniles contemporáneas (Reguillo, 2012).

Las nuevas generaciones, y sus consumos musicales, son en buena medida producto de tres grandes hitos tecnológicos que dieron paso a una nueva forma de conseguir, almacenar e intercambiar música: 1) Internet y el explosivo crecimiento de usuarios que se dio a partir de los años noventa; 2) La creación de tecnologías que permitieron la compresión en formato MP3, lo que generó el manejo digital de manera práctica y estandarizada, 3) La creación de Napster, programa pionero para el intercambio P2P de contenidos musicales entre los usuarios de la red (Sánchez, 2009, p. 13). Además de estos tres grandes hitos, el consumo de música vía streaming representa una nueva etapa de consumo musical. Como señala Pisani y Piotet (2009), según la dinámica relacional de la web 2.0, las personas no quieren tanto poseer la música físicamente como acceder, compartir y comentarla con otros.

La irrupción de las TIC a partir de los ochenta ${ }^{6}$ no solo produjo el fin de un modelo de negocio, también, como apunta Héctor Fouce, representaba un punto de quiebre: "lo que está en crisis no es un modelo de negocio, sino también un modelo de consumo y, por ende, un modelo de relaciones culturales" (2009, p. 65). El fin de este modelo coincidió con una generación de jóvenes cuyo entorno estaba definido por la cultura digital, individuos inmersos en lo que Perona, Barbeito Velozo, y Fajula llaman (2009) sono-esfera digital y que definen a partir de tres elementos básicos: a) un nuevo entorno caracterizado por la convergencia de los actuales medios digitales; b) nuevos hábitos de consumo por parte de los públicos; c) una variedad de dispositivos que relegan a la radio como principal emisor de contenidos sonoros. La convergencia digital sobre todo, como afirma Weinberger (2011), ha favorecido un conocimiento social, móvil e interconectado. 
La sono-esfera digital también promovió algo que Michael Bull $(2007,2010)$ ya había consignado: una suerte de esfera privada o burbuja sonora en donde los oyentes se sumergían en consumos privados y personalizados, gracias a todos aquellos dispositivos digitales portables que les permitían aislarse del entorno, construyendo su propio universo sonoro. Esta esfera sonora permitió a los jóvenes abrir posibilidades que en la era analógica no existían; decidir cómo, dónde, qué y cuánto escuchar; sonorizar su experiencia en el espacio público y organizar la música en función de actividades cotidianas diversas, sonorizadas con reproductores MP3 y los propios teléfonos móviles. Las TIC y su intensa presencia en las culturas juveniles, las han erigido en formas de mediación privilegiadas para el consumo musical.

\section{METODOLOGÍA}

Con el objetivo de comprender los consumos y usos sociales de la música de los jóvenes de la Zona Metropolitana de Querétaro, para la investigación se diseñó una estrategia metodológica de carácter cualitativo. El marco teórico evidenció la tendencia de los investigadores a darle un papel central y definitivo a Internet en los consumos juveniles de la música, lo cual suscitó la siguiente hipótesis de trabajo: Las TIC se convirtieron para las culturas juveniles en espacios hegemónicos de mediación, socialización, consumo y conformación determinantes del habitus musical. Con base en esta hipótesis, se optó por un estudio de caso, los jóvenes consumidores de la Zona Metropolitana de Querétaro (ZMQ), México, que comprende los municipios de: Querétaro, El Marqués, Corregidora y Huimilpan. Se eligió este caso por el interés en analizar los consumos musicales de los jóvenes que habitan esta geografía y que de forma escasa han sido estudiados.?

Las características de la población juvenil de Querétaro la hacen paradigmática para la comprensión de las culturas juveniles mexicanas. No solo por la importancia de la ciudad en el desarrollo nacional, ${ }^{8}$ sino por ser escenario de diferentes procesos de globalización y cruces interculturales en los que los jóvenes son protagonistas.

Querétaro se encuentra en la parte central de la República Mexicana, en una zona denominada "Bajío". Es uno de los seis estados más pequeños del país y está conformado por 18 municipios. Su cercanía con el Distrito Federal, capital del país, lo ha colocado en una posición geográfica privilegiada. A lo largo de la autopista denominada "México-Querétaro" se ha conformado un corredor industrial que alberga varios parques en donde operan empresas de diferentes giros, pero sobre todo pertenecientes a la industria automotriz y aeronáutica.

Esta actividad industrial ha provocado que la mitad de la población del estado se concentre en los municipios de carácter urbano cercanos a los parques industriales. Según cifras del INEGI (2014), la población total del Estado de Querétaro es de 1.827 .937 habitantes, de los cuales $887.188(48,5 \%)$ son hombres y $940.749(51,5 \%)$ mujeres. El municipio más poblado es Querétaro, que alberga a 801.940 personas, que representan el 43,9\% del total de la población del estado. De la población total, el 33,7\%, es decir 617.382 son jóvenes de entre 12 y 29 años. Del total de la población juvenil por grupos de edad: 108.321 entre 12 y 14 años; 187.241 entre 15 y 19 años; 168.520 de 20 a 24; 153.300 de 25 a 29 (INEGI, 2014).

En cuanto a educación, en Querétaro podemos ver que la mayoría de los jóvenes son estudiantes de secundaria y en menor número de preparatoria y universidad. En el estado, de cada 100 jóvenes, dos no han logrado incorporarse o permanecer en el sistema educativo, cinco tienen entre uno y cinco grados aprobados de primaria; 17 concluyeron la primaria; 38 
cuentan con algún grado de secundaria o estudios equivalentes; 24 tienen al menos un grado de estudios medio superior y 13 aprobaron algún grado de estudios superiores (INEGI, 2014).

Es importante saber para fines de esta investigación cuántos jóvenes tienen acceso a Internet en el estado de Querétaro en el contexto de la realidad mexicana. ${ }^{9}$ Aunque la Encuesta Nacional de Juventud (2010), en el apartado de Querétaro no contempla todas las formas de acceso a Internet, sí provee el dato de que el 65,4\% de los jóvenes tiene acceso desde su casa y saben usar este medio. En cuanto a entretenimiento, la principal actividad de los jóvenes queretanos entre 12 y 29 años, de acuerdo a la Encuesta Nacional de Juventud 2010 se muestra de la siguiente manera: reunirme con amigos 22,3\%, ver televisión 15,5\%, escuchar música $13,2 \%$, salir con mi pareja $11,8 \%$, ir al parque $6,5 \%$, ir a bailar $5,2 \%$, ir al cine $5,2 \%$, ir de compras 4,0\%, jugar video juegos 2,7\%, conectarme a Internet 2,1\%, leer 1,9\%. Cabe destacar que los jóvenes queretanos como actividad recreativa principal usan menos la conexión a Internet que el promedio nacional, que alcanza un 4,0\%, pero respecto a la escucha musical se encuentran por arriba del promedio nacional que es de 8,9\%.

Los jóvenes consumidores de música de la Zona Metropolitana de Querétaro están inmersos en su realidad local, pero expuestos a una cultura musical global compartida por jóvenes en todo el mundo. Si bien es cierto que las condiciones locales determinan en buena medida sus consumos musicales, existen procesos de hibridación, movilidad y globalización que convierten a estos jóvenes en consumidores que guardan importantes coincidencias con los de otras geografías o culturas. Con esto queremos subrayar que los casos de juventudes particulares mantienen cada día más vecindades y similitudes con otras de origen y localización distinta.

Empecemos por la dimensión global de la juventud, aspecto que debe de tomarse en cuenta cuando hablamos de casos específicos y localizados como el que nos ocupa. Ulrich Beck (2007) propone remplazar el concepto clásico de "generación", por el de "constelaciones generacionales transnacionales," en un intento de sugerir que aunque las diferentes realidades juveniles están fuertemente marcadas por lo local, hay un patrón cosmopolita que las influye. En este sentido los investigadores hablan de una generación actual que cuenta con estas tres condiciones: a) los procesos migratorios transnacionales, ${ }^{10}$ b) la precarización laboral, ${ }^{11}$ c) los procesos de hibridación cultural —en concreto por la proximidad a la "cultura mundo" (Lipovetsky y Serroy, 2010), potenciada por la hegemonía cultural del vecino Estados Unidos. De acuerdo con esto, puede decirse que la juventud actúa como un catalizador de tendencias emergentes y que incorpora a su realidad local una dimensión global, presente en sus prácticas en la vida cotidiana.

En la misma línea Feixa y Nilan (2006) sostienen que "juventud global"

Debe ser entendida como un colectivo híbrido — tanto a nivel local y mundial— que construye su subjetividad a partir de los materiales híbridos provistos por culturas, consumos, resistencias, tradicionalismos, digitalismos globales o globalizados. (2006)

En este sentido, consideramos que es importante el estudio de los consumos juveniles de música por parte de los jóvenes queretanos porque constituyen un buen ejemplo de jóvenes que, si bien es cierto construyen su relación con la música desde lo local, también atienden a los movimientos globales de música y forman parte de una "comunidad generacional" (atravesada por una cultura juvenil). 
Los procesos de globalización, y sobre todo de las TIC, generan en los jóvenes "hábitos culturales en función de bienes e imaginarios mundializados" (García Canclini, 2007, p. 6). También debemos considerar que con la llegada de Internet la juventud amplió sus horizontes y fronteras: además de los espacios naturales como el barrio o la ciudad a la que pertenece, el joven tiene a su alcance una cantidad de información que le ha permitido redefinir su individualidad y sus espacios colectivos.

Internet dejó un saldo positivo: el aumento de la percepción de la importancia de la virtualidad en la constitución de sujetos individuales y colectivos. Me refiero al papel de la imaginación en la constitución de una nueva colectividad, pero enfatizo el lugar de la virtualidad en este proceso. (...) La calidad de la relación entre el espacio-público-real y el espacio-público-virtual es un componente progresivamente importante para la construcción de cosmopolíticas en la contemporaneidad. (Ribeiro, 2003, p. 35)

Pretendemos entonces encontrar en este caso, sus especificidades, pero sin perder de vista que muchas de ellas estarán inscritas en consumos musicales globales, que pueden eventualmente encontrarse en otros jóvenes de culturas diferentes, pero con prácticas comunes derivadas del uso de Internet principalmente, y de las TIC en general. Además de ello, la industria mundial de la música —incluida la música en directo— es un elemento que continúa presente en el consumo de música juvenil, incluso desde antes de la irrupción de la música digital. Huelga decir que para poder saber cómo las dimensiones de lo local y lo global se cruzan, se necesitan estudios comparativos de consumos y prácticas juveniles en torno a la música.

Los jóvenes de nuestro grupo de estudio viven inmersos en una realidad que les plantea tensiones y problemáticas propias del momento por el que país atraviesa. Como la mayoría de los jóvenes en México, viven en un contexto urbano en el que el desempleo es una amenaza y en donde las nuevas tecnologías se han ido incorporando a su vida y a sus prácticas de consumo y entretenimiento.

Por lo antes visto, se considera a la Zona Metropolitana de Querétaro un importante escenario para estudiar fenómenos relacionados con sus jóvenes, como sus consumos y usos musicales. El papel protagónico de las culturas juveniles en la ciudad obliga a conocerlos a través de una investigación empírica, encaminada a recoger sus testimonios y declaraciones. Detallaremos en seguida nuestra estrategia metodológica.

La elección de los jóvenes estudiados se centró en aquellos consumidores consuetudinarios para quienes el consumo de música fuera una actividad significativa integrada a sus actividades cotidianas. Jóvenes que podían brindar información acerca de cómo se apropian de la música en el contexto de la era digital. Los sujetos de estudio fueron abordados en las plazas públicas que se encuentran en la Zona Metropolitana de Querétaro; con la condición de que estuvieran escuchando música a través de auriculares. Esta característica nos aseguró que los jóvenes eran consumidores de música, pues al llevar consigo reproductores MP3 habían seguido un proceso de consumo básico: la descarga a través de su ordenador y el almacenaje para su escucha final en su reproductor MP3. La muestra planteada obedeció a criterios conceptuales o teóricos, lo que nos acercó al tipo de muestreo denominado "juicio" o "muestra teórica" (Bernal, 2006). Es de carácter no probabilístico, pues se eligió a jóvenes considerados "expertos" de acuerdo a lo que la metodología cualitativa contempla, que en este caso fueron consumidores de música, y por ello son los sujetos de estudio de la investigación. 
De acuerdo a la Encuesta Nacional de Juventud 2010, El Estado de Querétaro, México, cuenta con un universo de 617 mil jóvenes, de los cuales el 48,7\% son hombres y el 51,3\% mujeres; la Zona Metropolitana de Querétaro, por su parte, cuenta con una población juvenil de 310.300 individuos. ${ }^{12}$ La muestra comprendió informantes que cumplían con las siguientes características: a) jóvenes de la Zona Metropolitana de Querétaro, b) jóvenes entre los 1529 años de edad, ${ }^{13}$ c) jóvenes consumidores de música. La muestra quedó integrada por 47 hombres y 24 mujeres para completar un total de 71 jóvenes; 41 individuos del municipio de Querétaro, 12 de Corregidora, 11 de El Marqués y 7 de Huimilpan; 44 de ellos de clase media, 14 de clase desfavorecida o baja, y 13 de alta. ${ }^{14}$

Se eligió el espacio público como lugar de encuentro con los informantes y como el centro de la praxis investigativa. Justificamos esta decisión desarrollando un concepto que denominamos geografías sociales, tomando como apoyo para ello propuestas de uso de espacio público propias de la geografía y la antropología urbana como Sidewalk de Mitchell Duneier (2001), estudio llevado a cabo en New York, en donde pretende fijar su mirada en la acera como el lugar donde se expresan muchas de las tensiones de la vida urbana, así como las variadas prácticas propias de las interacciones cotidianas.

Las plazas públicas de Querétaro tienen una gran importancia en el imaginario colectivo de sus habitantes, pues representan un espacio simbólico donde se encuentran los poderes político y religioso, y donde se llevan a cabo diferentes prácticas de socialización y entretenimiento popular. ${ }^{15}$ Elegimos para este estudio las plazas localizadas en el Centro Histórico del municipio de Querétaro: el Jardín Zenea, Jardín Guerrero, Plaza de Armas y municipio de El Marqués y el Jardín Central del municipio de Huimilpan.

La técnica elegida para obtener los datos fue la entrevista semi-estructurada, por considerarla el instrumento más idóneo para acceder a información de carácter cualitativo que nos permitiera entender de manera profunda las formas de apropiación musical de los jóvenes estudiados. Se utilizó además un diario de campo donde se registraron los aspectos que consideramos relevantes para describir, argumentar e interpretar los datos que se obtuvieron durante la fase de entrevistas (Martínez, 2007).

Derivado de la discusión teórica se creó un modelo analítico para trabajar con la información, tanto en la fase del diseño del guion de la entrevista, como del análisis de la información obtenida. Aunque el modelo incluyó dos conceptos, uno de ellos estaba dedicado a explorar funciones sociales de la música, e incluía las dimensiones identidad y expresión emocional; aquí solo explicaremos el que tiene que ver con el segundo concepto del modelo: usos musicales, el cual estaba destinado al análisis de las prácticas de consumo musical de los jóvenes, como precisamente lo expresa su primera dimensión llamada así, consumo; que a su vez se subdivide en los componentes "Prácticas de apropiación" y "Atributos de uso". Explicaremos - por la temática de lo aquí expuesto- solo el primero, que tenía como cometido identificar el lugar donde se realizaban las rutinas que involucraban el consumo musical y los procesos de mediación tecnológica implementados para consumo de música. Mediante este concepto e indicadores se realizó el diseño del guion de preguntas vinculadas con el tema y después sirvió para su respectivo análisis. 


\section{PRINCIPALES RESULTADOS}

Empezaremos por decir que aunque hay prácticas generalizadas de consumo, como el uso de Internet y la descarga de contenidos musicales a través de este medio, hay diferentes tipos y prácticas de apropiación que es importante señalar, pues representan matices y caracterizaciones que permiten comprender particularidades básicas para el entendimiento del papel de las TIC en las apropiaciones juveniles de música de los jóvenes de la muestra.

Observamos tendencias que se agrupan en los siguientes aspectos generales: a) la música es un elemento omnipresente en el cotidiano juvenil, que acompaña a los jóvenes a lo largo del día, tanto en actividades en espacios privados como públicos. b) a pesar de una tendencia a gustos variados o eclécticos, el rock es el género musical dominante entre los jóvenes estudiados, siendo los pares los principales prescriptores musicales, c) Internet se ha convertido en el medio más importante de consumo musical, no solo para la descarga, sino para múltiples usos, siendo YouTube la plataforma más utilizada, d) los teléfonos celulares son el dispositivo dominante en el consumo musical juvenil, desplazando a otro tipo de reproductores de MP3.

Desde la percepción de los jóvenes entrevistados, la música está incorporada plenamente a la rutina diaria, es parte de los espacios temporales, actividades y lugares que conforman su vida cotidiana, es según ellos la forma de entretenimiento más abundante y presente en sus vidas, el consumo cultural dominante. De acuerdo a su dicho, la música cobra una centralidad en su vida porque los acompaña en todo momento y en la variedad de actividades que realizan durante el día. ${ }^{16}$ La primera evidencia transversal es una omnipresencia de la música en el cotidiano juvenil. Los jóvenes que dicen oír música todo el día son en su mayoría hombres entre 20 y 25 años, que escuchan música en movimiento, en sus trabajos, y en horas de descanso en casa. ${ }^{17}$ "Los audífonos los traigo todo el día. Me gusta estarlos escuchando, ya sea que vaya caminando, y los vaya escuchando, vaya en el camión, o esté aquí sentado, igual los escucho" (E26, M, 23 años, Jardín Guerrero). ${ }^{18}$ La disponibilidad de equipos y el uso de auriculares los provee de una música omnipresente que los acompaña sin importar el lugar y el momento.

La omnipresencia de la música logra incluso la musicalización total del día a día de los jóvenes. El día musicalizado solo es posible por la escucha móvil que las nuevas generaciones realizan principalmente desde sus teléfonos celulares, dejando atrás otro tipo de gadgets o dispositivos. Esta omnipresencia de la música se conformó en la persistencia de consumos concretos que importa detallar en dos puntos fundamentales: el primero de ellos es la cultura juvenil atravesada por un uso hegemónico de la herramienta generacional; desde los discursos recogidos percibimos la centralidad de YouTube como acto performativo, inclusivo en una cultura juvenil. En segundo lugar, a raíz de la detallada portabilidad, detectamos la presencia de la música como banda sonora de unos jóvenes que viven bajo la era de la saturación cultural. La música se revela como un recurso emocional que potencia la imaginación y neutraliza el efecto desgastante de las rutinas cotidianas.

La música cambia la percepción de lugares y personas. Los jóvenes narran experiencias en este sentido, en donde la música los lleva a experimentar de manera diferente sus recorridos por la ciudad. "Yo lo veo así, o sea como si dijeran vas a hacer un video de música, pero ya con las personas que llego a ver. O sea, o tratándolo de interpretar con la misma canción, la misma gente, las mismas caras". (E41, M, 29 años, Plaza de Armas). Existe una relación entre la música y la dinámica al caminar por la ciudad, los jóvenes entrevistados reconocen su influencia en la manera como viven la experiencia del tránsito urbano. 
Comprobamos con nuestro estudio lo que afirma de manera teórica Chambers (1985): Ios jóvenes crean una identidad auditiva nómada a partir de la escucha en movimiento. La escucha móvil ayuda a los jóvenes de nuestra investigación a acompañar su cotidianidad mediante un pautado musical en donde los dispositivos móviles son muy importantes. Esta omnipresencia como práctica continuada juvenil parece poder resumir de forma clara la evidencia mayor de este estudio: que la música es el elemento social más expresivo de las culturas juveniles actuales.

La rutina musicalizada está indefectiblemente vinculada al gusto musical, los jóvenes escuchan casi todo el día aquella música que eligen de acuerdo a sus preferencias, por ello vale la pena analizar la naturaleza de su gusto musical. Si bien es cierto que las escuchas de los jóvenes se nutren de varios géneros musicales, el rock es un género dominante en el gusto de la mayoría de jóvenes de la muestra, aunque se ve dividido en subgéneros, la preferencia por esta expresión musical es clara: “De la música que me gusta más que nada el rock, y me gusta el metal, el rock antaño de los 80 y 90. Todo lo que se escucha en Mix, así como Bee Gees, clásicos, de los Beatles, de los Credence, Kiss, me gusta todo ese tipo de música" (E26, M, 26 años, Jardín Guerrero).

Los principales prescriptores musicales para la mayoría de los jóvenes entrevistados son los amigos y su influencia tiene mayor impacto en la época adolescente entre los estudiantes de enseñanza media. "Empecé con lo que es alternativo, rock alternativo y cosas así. Comencé por un amigo que me enseñó una banda post-hardcore, y con esa me empecé a meter más a ese tipo de cosas. Entonces se podría decir que fue por una recomendación" (E11, M, 17 años, Plaza de Armas).

Pudimos concluir, en virtud de los testimonios de los entrevistados, que las principales influencias para la construcción del gusto juvenil son los pares, no la información obtenida en Internet. De tal manera que este medio es usado como una herramienta para conocer más o conseguir aquello referido por los pares en relaciones interpersonales cara a cara. En este sentido las estructuras sociales fuera de Internet, como los pares y la familia, siguen jugando un papel preponderante en la construcción del gusto musical. Podemos decir que la esfera digital es una extensión de un proceso de socialización musical previo, brindado por los grupos fuera de Internet a los que los jóvenes pertenecen. Según esta lógica, pudimos comprobar que los jóvenes utilizan Internet como un filtro musical o una herramienta de consumo, con la que prueban y se informan acerca de lo dicho por sus pares en situaciones de interacción no mediada por Internet.

A partir de la evidencia empírica, podemos decir que Internet cambió la noción de la música como producto, convirtiéndola en información. Esto liberó a los consumidores de su papel y dependencia de las disqueras, para convertirlos en oyentes que acceden a la música gratuitamente y de manera natural, pues el nuevo paradigma ha cambiado, difuminando los papeles de ofertante, distribuidor y consumidor. Lo más importante de este cambio es que el joven dejó de ser un comprador para convertirse en un oyente, cuya lógica no es la de la adquisición a través de un mercado musical, sino la de un escucha frente a un gran bazar digital llamado Internet, en donde puede conseguir música sin formar parte de la estructura y proceso de compraventa de la misma. Las consecuencias más directas de esto son la hiperabundancia y la eliminación del concepto de posesión de la música y del derecho de autor.

Dentro de la dimensión consumo se incluyó el tema de cómo consiguen ${ }^{19}$ los jóvenes la música. Ellos utilizan la descarga como la base de sus consumos. La descarga permite la 
variedad de géneros, la abundancia de música y el acceso rápido y gratuito; si y sólo si al joven le gusta mucho un material, lo compra, reduciendo esta forma de consumo a un suceso eventual o extraordinario, pues la mayor parte de su música viene de las descargas habituales. "Descargo y pues si veo un CD que está bueno, pues lo compro" (E43, F, 20 años, Huimilpan).

La descarga es la práctica principal de los jóvenes a la hora de conseguir la música; Internet cuenta con una diversidad de páginas Web, plataformas y software para ello. Por tanto, se interrogó a los miembros de la muestra acerca de los recursos que utilizaban para su consumo y las razones de su uso. La primera gran tendencia en el uso de plataformas digitales fue YouTube, ${ }^{20}$ a la que la mayoría de los jóvenes — tanto hombres como mujeres- recurren no solo para informarse acerca de artistas o novedades, o para descargar videos, sino para convertir estos en archivos de audio que puedan descargar e incorporar a sus bibliotecas musicales. Este giro en el uso de YouTube representa un gran impacto en las prácticas de consumo de música de los jóvenes, pues estos están migrando de los buscadores tradicionales a esta plataforma que se ha ido convirtiendo en la central de información y descarga para los consumidores de música. "Normalmente siempre las descargo de YouTube, hay una página especial para bajar los videos que sube la gente, pues puedes bajar solamente el audio, así MP3" (E8, M, 19 años. El Marqués).

En cuanto al equipamiento, durante el periodo de entrevistas se tuvo la oportunidad de interrogar a los jóvenes acerca del hardware a su disposición, e incluso de observar directamente en el trabajo de campo los equipos móviles con los que los jóvenes contaban. ${ }^{21}$ Computador y teléfono celular constituyen el equipamiento mínimo que detectamos, es el esquema básico para acceder a la música digital y luego reproducirla de manera móvil. El tándem computadorteléfono celular es el resultado de la simplificación y economía de espacio y recursos de los jóvenes. Muchos de los usuarios de este formato declaraban no necesitar más, teniendo a su teléfono como reproductor único de su música. El teléfono móvil ha desplazado paulatinamente a los reproductores de MP3, sobre todo entre los más jóvenes.

La multifuncionalidad de los teléfonos celulares constituye un elemento central en su hegemonía como dispositivos preferidos de los jóvenes. Un teléfono celular ${ }^{22}$ es la gran herramienta que el joven tiene para la comunicación, la interacción con sus pares y el manejo de sus archivos musicales. Hoy en día los jóvenes aspiran a la posesión de teléfonos inteligentes con los que puedan hacer todo lo que antes hacían con dos o hasta tres gadgets: "Con este teléfono yo ahorro, porque en eso de fotografía, me ahorro lo de la cámara porque este trae una, y aparte también puedo reproducir bien mi música y ya traigo muchas cosas ahí, para mi uso" (E22, M, 16 años, Jardín Zenea).

Para resumir lo que tiene que ver con equipamiento, podemos decir que hay una clara tendencia a reducir el número de equipos a la fórmula computador-teléfono móvil; el computador usado para la descarga y escucha doméstica, y el teléfono como centro de comunicación y entretenimiento móvil. En cuanto a los reproductores MP3, los iPods quedan reservados para consumidores de música más especializados que pretenden tener grandes acervos, o que reconocen a sus reproductores como los contenedores de sus colecciones personalizadas. Los reproductores MP3 como equipos principales están siendo usados por jóvenes de clase media, con un mayor ingreso y rango de edad, pues los más jóvenes se han iniciado en la escucha de música digital básicamente con el teléfono como primer equipo, sin vivir la transición de un gadget a otro. 


\section{CONCLUSIONES}

El presente estudio, que ahonda en las prácticas de la apropiación musical de los jóvenes de Querétaro, se suma a los escasos intentos empíricos que pretenden comprender el papel de las nuevas tecnologías en el consumo juvenil de música. No obstante su carácter exploratorio, nos permite barajar algunas conclusiones acerca de las apropiaciones musicales de los jóvenes en un contexto marcado por los usos intensivos de las TIC.

En línea con las investigaciones que señalamos a lo largo de este artículo, la tecnología atraviesa el cotidiano juvenil de forma omnipresente. Los consumos culturales juveniles están marcados de forma contundente por la mediación digital, derivada de un proceso de socialización pautado por Internet que ayudó a conformar prácticas y gustos culturales juveniles masificados y generadores de una fuerte identidad pop. Las tecnologías en red contribuyeron a conformar lo que Lipovetsky y Serroy (2010) nombraron cultura-mundo, lo que hace de los jóvenes de Querétaro arquetipos de consumidores, con potencial heurístico suficiente para percibir una determinada práctica generacional.

La interpretación de nuestros datos, a la vez que aportan validez a esta tesis dominante, propone interesantes pistas que desmitifican conclusiones tajantes y normalizadoras sobre los consumos musicales juveniles. En sintonía con resultados obtenidos con otros estudios, verificamos que la música se asumió ante todo como un contenido destituido de su sentido de producto. Varias evidencias parecen contribuir para esta conformación. Internet, desde sus distintas plataformas, contribuyó a desmaterializar el artefacto musical. La cultura streaming (muy alimentada por YouTube) y de download (legal o ilegal), actividad cotidiana de todos los jóvenes entrevistados por nosotros, modificó la relación del sujeto con el soporte musical. ${ }^{23}$

Esta destitución de la corporeidad del objeto artístico, a la par de la posibilidad "gratuita" de un acceso cotidiano gigantesco a contenidos musicales, ha hecho que los jóvenes internalicen sus consumos musicales como derivados de una naturalización del uso de Internet, lo que diluyó fuertemente el concepto de derecho de autor.

Esta intensa y continua relación de los consumos musicales mediados por las TIC asume un eje central en la relación social de los jóvenes, ya que la música es concebida como un fuerte elemento de sociabilidad para ellos, en particular con sus grupos de pares. Al igual que en otros estudios ya mencionados, registramos que los amigos y colegas asumen un papel vital en los consumos musicales efectuados (con una fuerte preponderancia en la conformación de los gustos y de los oyentes musicales). Registramos una tendencia para una cultura juvenil de consumo heterogéneo (en cuanto a géneros y tendencias sonoras) muy influidas por fenómenos que surgen y discurren a través de los mass media, que contribuyen para una escucha muy marcada por un proceso de volatilidad y fragmentación del gusto (se escucha el artista de moda, el hit de turno que amplifica su valor social en los consumo mayoritarios).

De los discursos extraemos el papel central de la música en el rol determinante que ocupa en una cultura juvenil fuertemente visual y performativa (YouTube es la plataforma con mayor preponderancia en los consumos). La construcción del self se perfila colectivamente por los procesos de identificación que los jóvenes conforman desde los referentes estéticos (estilos y modas), emocionales (los modos de ser de los artistas) e identitarios (tomas de posición políticas, opiniones de corte social).

$\mathrm{Si}$ estos aspectos conforman los atributos de una cultura-mundo que configuran una determinada manera generacional de apropiación musical de los consumos, registramos un 
conjunto de otras pistas que nos abren una disyuntiva en las prácticas de estos jóvenes. Importa señalar algunas asimetrías verificadas, que matizan aspectos que no vimos registrados en las aportaciones empíricas revisadas por nosotros. Registramos usos desiguales en relación con los consumos musicales de los jóvenes provenientes de clases sociales más desfavorecidas (una de las dimensiones que tuvimos en cuenta a la hora de la recolección de datos).

La cuestión central está determinada por el acceso a Internet, que altera de manera profunda la forma como los jóvenes se relacionan con los contenidos musicales. Observamos comparativamente los usos de clases bajas con las clases medias/altas y registramos que la falta de autonomía de los primeros (privados del acceso de Internet en casa) genera que adopten como estrategia solicitar sus descargas en el ciber en turno.

La dependencia de la fórmula radial y una homogenización de los géneros escuchados (rock, pop, reggaetón, banda) impone un fuerte efecto de gatekeeping reduciendo el abanico de opciones estéticas y culturales que propone la cultura-mundo. La conformación de determinados consumos musicales, marcados por referentes de un cariz más local y culturalmente menos sofisticados, contribuye para una doble exclusión. Por un lado son estigmatizados por los consumidores de la cultura-mundo y por otro lado, la música que escuchan no sirve de referente para potenciar otros imaginarios culturales.

Los consumidores de la cultura-mundo, en sus deambulaciones por el ciberespacio expanden sus horizontes y referentes culturales. La búsqueda incesante que parte de las sugerencias expandidas de sus grupos de pares (de forma más escasa de su familia) o de los mass media (y aquí el plural marca toda la diferencia), convierte la escucha en un entretenimiento pedagógico.

En resumidas cuentas, y recuperando la pregunta central de esta investigación, los actuales consumos musicales juveniles presentan renovadas formas de apropiación mediante la utilización intensiva de las TIC. Este hecho merece, sin embargo sus debidos matices, pues existen brechas digitales derivadas del género, la clase social y la alfabetización tecnológica que determinan los usos, formas de acceso y la relación misma de los jóvenes con la música.

\section{NOTAS}

1 El término fue acuñado por Richard Hoggart, al fundar el Centro de Estudios Culturales Contemporáneos (Centre for Contemporary Cultural Studies) en Birmingham.

2 Los principales estudios sobre subculturas juveniles presentan una diversidad de temas, pero entre los que están relacionados más directamente con música y estilos vale la pena mencionar los trabajos de Stanley Hall y Tony Jefferson, Resistence thorough rituals (1975); Paul Willis, Learning to labor (1977) y Disck Hebdige, Subculture (1979) de la escuela inglesa. Michel Maffesoli, El tiempo de las tribus: el declive del individualismo en las sociedades de masas (2004), y los de la canadiense Sarah Thornton, Club Cultures, (1996); Steven Miles, Youth lifestyles in a changing Word (2000), de la escuela postsubculturalista. Más recientemente los trabajos de Maffesoli, La trasformación de lo político, la tribalización del mundo posmoderno (2005), así como Iconologías, nuevas idolatrías posmodernas (2009).

3 Para mayor información sobre este tipo de estudios se recomienda consultar los trabajos desarrollados por la Universidad Macquarie (Australia), que cuenta con Music and Sound Cultures, departamento orientado a la investigación sobre la música como objeto de estudio.

4 Destacan otros trabajos en España orientados a recuperar el estudio de la música desde la comunicación. Selva, D (2014). El videoclip. Comunicación comercial en la industria de la música. Sevilla: Ediciones Alfar. Revista Comunicar, número especial Comunicación, Música y nuevas tecnologías. 
5 A la música popular la podemos definir como: "Aquella música contemporánea de tradición no clásica, que utiliza instrumentos de amplificación electrónica y que está orientada principalmente al público juvenil" (Pitarch y Ellis, 2005: 12).

6 Martín Varsavsky (2007) reconoce tres etapas en la industria de la música en los últimos treinta años: 1.- años ochenta: aparición de la tecnología digital. 2.- años noventa: creación del formato MP3. C.- primera década del siglo XXI: masificación de la banda ancha y web 2.0.

7 Cabe señalar que a pesar de sus ventajas, los estudios de caso tienen una limitación inherente a su naturaleza, pues los resultados se limitan a explicar una realidad concreta y particularizada.

8 La ciudad de Querétaro está posicionada como una de las principales ciudades para hacer negocios y con mayor potencial financiero en Latinoamérica (IMercer,20), (Financial Times, 2015; KPMG 2015; ARegional, 2013).

9 De acuerdo al $11^{\circ}$ Estudio sobre los Hábitos de los Usuarios de Internet en México 2015, realizada por la Asociación Mexicana de Internet (AMIPCI), en México existen para el año 2014 53,9 millones de usuarios, lo cual representa un incremento del 18,3\% respecto al 2012. Para el 2015 el porcentaje de población que utiliza Internet entre hombres y mujeres presenta un porcentaje igualitario, es decir, $50 \%$ en hombres y $50 \%$ para mujeres. Otro dato que se ha disparado respecto al 2014, es el nivel de conexión, que actualmente es de 6 horas y 11 minutos, 35 minutos más que en la encuesta anterior (AMIPCI, 2014). Dentro de las principales actividades en línea del internauta mexicano están el acceder a Redes Sociales (85\%), buscar información (78\%) y enviar y recibir emails (73\%). (AMIPCI, 2015). Respecto a las principales actividades de entretenimiento en línea del internauta mexicano está de nuevo el uso de Redes Sociales (83\%) utilizado principalmente por mujeres de entre 19 y 24 años, y el enviar y recibir emails (55\%) presentándose en hombres y mujeres de más de 19 años. Las principales redes sociales en México son Facebook, Twitter e Instagram respectivamente (AMIPCl, 2015). El volumen de usuarios de Internet en México crece año con año, en el 2012 el país ocupaba ya el lugar número once de volumen de internautas en el mundo (WIP, 2013), y la mayoría de los estudios realizados sobre preferencias y hábitos de consumo de Internet en México consignan que los jóvenes son el grupo mayoritario de usuarios de la red. Tal es el caso del estudio de medición de tráfico en la red "Futuro digital. México 2015" llevado a cabo por la empresa ComScore (2015), que reveló que el $52 \%$ de los internautas son jóvenes, cuyas edades oscilan entre los 15 y 34 años. El estudio muestra que las principales categorías de contenidos pertenecen en servicios a Google, Outlook y Yahoo con un 95\% de alcance, en entretenimiento Youtube y iTunes representan un 94\% y en Social Media Facebook, Blogger y Linkedln cuentan con un $91 \%$ de alcance. El Instituto Nacional de Geografía y Estadística (INEGI) de México, también coloca a los jóvenes como el principal grupo de usuarios de Internet. Este instituto considera que el 62,6\% de los usuarios de Internet en México son jóvenes entre 12 y 34 años. El principal uso que se da a la computadora en México es para obtener información de carácter general $(67,4 \%)$, seguido del acceso a redes sociales $(39,6 \%)$, actividades vinculadas con la comunicación (38,5\%), y actividades escolares $(36,7 \%)$. También se destaca que en números absolutos 34,8 millones de mexicanos usan computadora de escritorio, 16,4 millones utilizan computadora portátil (laptop) y 9,4 millones utilizan smarthphone (INEGI, 2014).

10 La tasa migratoria en Querétaro es de 3.4. Querétaro ocupa el $6^{\circ}$ lugar en cuanto a migración a nivel nacional, según datos de INEGI. Tomado de http://www.inegi.org.mx/prod_serv/contenidos/espanol/bvinegi/productos/ integracion/estd_perspect/qro/pers-qro.pdf.

11 De acuerdo a INEGI, en Querétaro hay 120 mil jóvenes que no estudian y no trabajan. Tomado de http://www. imjuventud.gob.mx/imgs/uploads/2_ENJ_2010_-_Queretaro_VF_Mzo_13_MAC.pdf

12 El Municipio de Querétaro 229.354; El Marqués 34.937; Corregidora 36.054; Humilpan 9.955.

13 Criterio etario usado por el Instituto de Geografía y estadística de México que considera jóvenes a los sujetos en este rango de edad.

14 En el guion, además de los temas relacionados con las dimensiones, se incluyeron preguntas cuyo objetivo era conocer las variables sociodemográficas de los jóvenes de la muestra. Para tal efecto se preguntó la edad, lugar de residencia, ocupación y actividad laboral de sus padres. Esto nos permitió inferir la clase social a la que pertenecen, así como contar con los datos básicos para el análisis de sus respuestas.

15 Las plazas del centro de Querétaro no son sólo un lugar de tránsito, también son espacios culturales que albergan conciertos, exposiciones y diferentes expresiones de orden político. Son el punto de encuentro de diferentes clases sociales, de locales y turistas, de vecinos y visitantes ocasionales. La concentración de servicios turísticos y de oferta de entretenimiento, preferentemente juvenil, las ha convertido en un lugar de paseo, encuentro y tránsito para diferentes clases sociales y estilos de vida. Por su importancia, estas plazas 
ocupan un lugar especial en el imaginario colectivo de los queretanos, en la cobertura mediática, e incluso en la investigación con fines académicos

16 La música para los jóvenes es un elemento muy importante en su cotidianeidad, pues está presente en diversas situaciones y por amplios espacios temporales (Green, 1997; Rabaioli, 2002; Gasperoni, Marcon y Santoro, 2004; North, Hargreaves y O'Neil, 2000; Frith, 1999).

17 Los jóvenes que no están dentro de esta tendencia consumen entre dos y cuatro horas al día, principalmente cuando realizan tareas o actividades escolares.

18 Las referencias a las entrevistas están etiquetadas de la siguiente manera: número de entrevista, género del entrevistado, edad, y nombre de la plaza o municipio donde fue realizada la entrevista. Cuando se trata de las plazas del Municipio de Querétaro, por ser cuatro diferentes espacios ubicados todos en su centro histórico, se utilizan el nombre de las plazas o jardín (Jardín Guerrero, Jardín Zenea, Plaza de Armas, Plaza Constitución). En el caso de los municipios más pequeños (en cuanto a superficie y habitantes) que conforman la Zona Metropolitana de Querétaro, al utilizar solo su plaza principal, se usa el nombre del municipio, para así diferenciarlos de las plazas del municipio de Querétaro. La referencia en este caso se remite a los nombres de los municipios: Corregidora, El Marqués, o Humilpan.

19 Debido a las diferentes formas para hacerse de ella, se utilizó la palabra conseguir y no comprar, en el entendido de que las prácticas relacionadas con ello iban desde la descarga hasta el intercambio; consiguiéndola legal o ilegalmente, y utilizando archivos digitales o discos físicos.

20 "YouTube continúa siendo el servicio de video en streaming más popular del mundo y cuenta con más de 800 millones de usuarios activos por mes. De los diez videos más vistos en la historia de este servicio, nueve son contenidos musicales. En noviembre de 2012, la canción Gangnam Style de PSY superó al tema Baby de Justin Bieber y se convirtió en el video más visto en YouTube tras registrar más de mil millones de visitas a tan solo cinco meses desde su fecha de lanzamiento" (IFPI, 2013, p. 16).

21 Durante las entrevistas pudimos ver los equipos usados por los jóvenes. La mayor parte de los jóvenes de la muestra escuchaban música desde sus teléfonos móviles, la mayoría de ellos smartphones de diversas marcas. Una minoría continúa usando reproductores MP3, sobre todo del modelo iPod. (Diario de campo, julioagosto del 2013).

22 En el trabajo de campo se pudo observar el amplio uso de teléfonos inteligentes entre los jóvenes. Incluso en los de menores ingresos se observó la posesión de teléfonos con la posibilidad de tomar fotografías, descargar contenidos y gestionar redes sociales.

23 El aura del objeto, en la acepción de Walter Benjamin, desapareció para esta generación de nativos cibernéticos que consumen música en formatos digitales. El sentido del objeto musical (vinilo, CD, DVD) es alimentado por toda una generación de nostálgicos socializados en el consumo musical pre-cibernético, una industria que fomenta por eso la retromanía, como argumenta Simon Reynolds (2012).

\section{REFERENCIAS}

Adorno, Theodor W. Filosofía de la nueva música. España: Akal, 1949.

Asociación Mexicana de Internet AMIPCI (2015). 11 Estudio sobre los hábitos de los usuarios de internet en México 2015. Recuperado de: https://www.amipci.org.mx/images/AMIPCI_ HABITOS_DEL_INTERNAUTA_MEXICANO_2015.pdf (Acceso: 28 de agosto de 2015).

Asociación Mexicana de Internet AMIPCI (2014). Estudio sobre los hábitos de los usuarios de internet en México 2014. https://www.amipci.org.mx/estudios/habitos_de_internet/Estudio_ Habitos_del_Internauta_Mexicano_2014_V_MD.pdf (Acceso: 28 de agosto de 2015).

Bernal, César. Metodología de la investigación. México: Pearson/Prentice Hall, 2006.

Bourdieu, Pierre. La distinción. Criterio y bases sociales del gusto. Madrid: Taurus, 1988.

Bull, Michael. “iPod: un mundo sonoro personalizado para sus consumidores". En Revista comunicar. Vol. XVII, núm. 34 (2010). 55-63 doi: 0.3916/C34-2010-02-05.

Bull, Michael. Sound Moves. iPod Culture and Urban Experience. London: Routledge, 2007. 
Calvi, Juan Carlos. "La industria de la música, las nuevas tecnologías digitales e Internet. Algunas transformaciones y salto en la concentración". En Revista de Estudios de Comunicación. España: Universidad del País Vasco. Vol. 11 (noviembre 2006): 121-137.

Carriço Reis, Bruno. "Cultura intangible ¿Una nueva materialidad del arte?" Artecontexto. [En línea] No. 22, 2009, pp. 7-9. http://www.artecontexto.com/en/readonline-22.html (Acceso: 20 de septiembre de 2014).

Chambers, lain. Urban Rhythms: Pop Music and Popular Culture (Communications \& Culture). USA: Palgrave Macmillan, 1985.

De Aguilera, Miguel, Adell, Joan E. y Sedeño, Ana. Comunicación y música II. Barcelona: UOC Press, 2008.

De la Peza, María Del Carmen. El bolero y la educación sentimental en México. México: UAM, 2001.

De la Peza, María Del Carmen. El Rock Mexicano, un espacio en disputa. México: UAM, 2015.

DeNora, Tia. Music in Everyday Life. Cambridge: Cambridge University Press, 2000.

Duneier, Mitchell. Sidewalk. EUA: Farrar, Straus and Giroux, 2001.

“Encuesta Nacional de Juventud 2010. Resultados generales". IMJUVE, 11 de Noviembre 2011. Imjuventud.http://www.imjuventud.gob.mx/imgs/uploads/2_ENJ_2010_Queretaro_VF_Mzo_ MAC.pdf (Acceso: 15 de enero 2014).

Finnegan, Ruth. "¿Por qué estudiar la música? Reflexiones de una antropóloga desde el campo". En Revista Transcultural de Música. [En línea] 6 (junio 2002), Barcelona. http://www. sibetrans. com/trans (Acceso: 28 de septiembre de 2014).

Fouce, Héctor. "Música digital, mundo global: la batalla por la tecnología de la música en Internet". En Actas del congreso. La ciencia ante el público. Salamanca: Universidad de Salamanca, 2002.

Fouce, Héctor. "No es lo mismo: audiencias activas y públicos masivos en la era de la música digital." En Comunicación y música 2. Tecnologías y audiencias. Barcelona: UOC Press, 2008. 111-134.

Fouce, Héctor. Más allá de la crisis de la industria discográfica: redes $P 2 P$, música y experiencia cultural generacional. Madrid: Universidad Complutense de Madrid, 2009.

Frith, Simon. "Hacia una estética de la música popular". En Las culturas musicales. Lecturas en etnomusicología. Madrid: Trotta, 2001. 413-436.

Frith, Simon. Sociología del Rock. Madrid: Ediciones Jucar, 1980.

Frith, Simon, Will Straw y John Street. La otra historia del rock. Aspectos clave del desarrollo de la música popular: desde las nuevas tecnologías, hasta la política y la globalización. Barcelona: Robinbook, 2006.

Financial Times (2015) "Global cities of the future 14/15. The winners", fDIntelligence, London. http:// www.fdiintelligence.com/Locations/Global-Cities-of-the-Future-2014-15- the-winners (Acceso: 29 de septiembre de 2014).

García Canclini, Néstor. Lectores, espectadores e internautas. Barcelona: Gedisa, 2007.

Gasperoni, Giancarlo, Marcon, Luca y Santoro, Marco. La musica e gli adolescenti: pratiche, gusti, educazione. Torino: EDT, 2004.

Green, Anne-Marie (Ed.). Des jeunes et des musiques: rock, rap, techno. Paris: L'Harmattan, 1997.

Hall, Stuart y Jefferson, Tony (Eds.). Resistance through Rituals: Youth Subcultures in Postwar Britain, London. New York: Routledge, 1976.

Hebdige, Dick. Subculture. The meaning of style. EUA: Routledge, 1979.

Lipovetsky, Gilles y Serroy, Jean. La cultura-mundo. Barcelona: Anagrama, 2010.

Lull, James. "The social uses of television." Human Communication Research Oxford, No. 6 (March 1980): 197-209. 
Maffesoli, Michel. El tiempo de las tribus, el ocaso del individualismo en las sociedades posmodernas. México: Siglo XXI, 2004.

Maffesoli, Michel. Iconologías, nuevas idolatri@s posmodernas. Barcelona: Península, 2009.

Maffesoli, Michel. La trasformación de lo político, la tribalización del mundo posmoderno. México: Herder, 2005.

Martínez, Luis A. "La Observación y el Diario de Campo en la definición de un tema de Investigación”. En Perfiles libertadores. Bogotá: Institución Universitaria Los Libertadores, 2007.

Merriam, Alan P. The Anthropology of Music. Illinois: Northwestern University Press, 1964.

North, Adrian C., Hargreaves, David J. y O'Neil, Susan A. (2000). "The Importance of Music to Adolescents". British Journal of Educational Psychology, London, V.70, Issue 2 (June 2000): 255-72.

Noya, Javier, Del Val, Fernán y Muntanyola, Dafne. “Paradigmas y enfoques teóricos en la sociología de la música". Revista Internacional de Sociología (RIS), España, Vol. 72, núm. 3 (septiembrediciembre 2014): 541-562.

Perona, Juan José, Barbeito Veloso, María L. y Fajula, Anna. “Los jóvenes ante la sono-esfera digital: medios, dispositivos y hábitos de consumo sonoro", Communication \& Society / Comunicación y Sociedad, Vol. 27, núm. 1, (2014): 205-224.

Pisani, Francis y Piotet, Dominique. La Alquimia de las multitudes: cómo la web está cambiando el mundo. Barcelona: Paidós, 2009.

Rabaioli, Inácio. "Práticas musicais extraescolares de adolescentes: um survey com estudantes de Ensino Médio da cidade de Londrina/PR". [En Línea] Tesis de Maestria, Porto Alegre, Instituto de Artes, Universidade Federal do Rio Grande do Sul, 2002. http:// www.biblioteca.ufrgs.br/ bibliotecadigital/2003-1/tese- -art-0377784.pdf (Acceso: 5 de Marzo de 2006).

Reguillo, Rossana. "Navegaciones errantes. De músicas, jóvenes y redes: de Facebook a Youtube y viceversa". En Nueva época. Guadalajara, No. 18, (julio-diciembre 2012): 135-171.

Rodríguez Suso, Carmen. Prontuario de musicología. [En línea] España: Clivis, 2008. http://www. clivis-music.com/llibres/prontuario.pdf (Acceso: 3 de octubre 2014).

Sánchez, Antulio. MP3, Internet y fin de los discos compactos. México: UAM, 2009.

Simmel, George. Estudios psicológicos y etnológicos sobre música. México: Editorial Gorla, 2003.

Steingress, Gerhard. "La música en el marco del análisis de la cultura contemporánea". En Política y sociedad. Madrid, No. 45, (2008): 237-260.

Weinberger, David. Too big to know. New York: Basic Books, 2011.

\section{Cómo citar este artículo:}

Rivera Magos, Sergio. y Carriço Reis, Bruno. "Los consumos juveniles de música en la era digital: un estudio de caso en la Zona Metropolitana de Querétaro". Cuadernos de Música, Artes Visuales y Artes Escénicas, 10(2), 171-192, 2015. http://dx.doi. org/10.11144/ Javeriana.mavae10-2.cjmd 